\title{
Comparison on the Use of Spinal (Stylet) Needle and Simple Needle in Ultrasound Guided Thyroid Nodule FNA; Does the Needle Affect Thyroid FNA Result?
}

\author{
Alireza Aziz Ahari ${ }^{1}$, Mohammad Ali Mohammadi Vajari ${ }^{1}$, Navid Khatibi Moghadam ${ }^{1}$, Hassan \\ Hashemi $^{2}$, Mahmoud Parvin ${ }^{3}$ and Mohammadreza Khaleghi ${ }^{1, *}$ \\ ${ }^{1}$ Department of Radiology, Hazrat Rasoul Akram Hospital, Iran University of Medical Sciences, Tehran, Iran \\ ${ }^{2}$ Advanced Diagnostic and Interventional Radiology Research Center (ADIR), Tehran University of Medical Sciences, Tehran, Iran \\ ${ }^{3}$ Department of Pathology, Shaheed Labbafinejad Hospital, Shahid Beheshti University of Medical Sciences, Tehran, Iran \\ "Corresponding author: Department of Radiology, Hazrat Rasoul Akram Hospital, Niyayesh St., Shahrara, Postal Code: 14456-13131, Tehran, Iran. Tel: +98-9128589226, Email: \\ mkh712003@yahoo.com
}

Received 2019 October 08; Revised 2020 February 05; Accepted 2020 March 21.

\begin{abstract}
Background: Thyroid fine needle aspiration (FNA) is currently the most acceptable method for evaluating thyroid nodules. Nonsatisfactory sample is the main limitation of FNA. It has been shown that spinal needles can reduce the number of non-diagnostic samples.

Objectives: In this study, we decided to compare the results obtained using spinal (stylet) needle and simple needle in thyroid nodule FNA according to the agreement between them and with the pathology results as the gold standard.

Patients and Methods: Sampling of thyroid nodules of 156 patients was performed by a simple non-stylet 23 gauge needle (A) and a spinal 22 gauge needle with stylet (B). The samples were provided to the pathologist for blind examination. All samples were obtained by an expert radiologist and the pathology examination was also performed by a pathologist expert in thyroid gland diseases. Blind analysis was done using SPSS Statistics for Windows, version 16 (SPSS Inc., Chicago, Ill., USA).

Results: The results of FNA with needles A and B were reported unsatisfactory in 11.51 (18 subjects) and 3.84 (six subjects), respectively $(\mathrm{P}=0.01$ ). The result in $63.46 \%$ (99 subjects) of the participants, in whom FNA was obtained by needle B was introduced as a better sample by the pathologist in comparison with $36.44 \%$ (57 subjects) $(\mathrm{P}<0.001)$. Cancer was diagnosed in 9.56 and 7.66 of the samples yielded by needles $\mathrm{B}$ and $\mathrm{A}$, respectively, which was not statistically significant $(\mathrm{P}=0.54)$. There was also a significant relationship between thyroid imaging reporting and data system (TI-RADS) score and FNA result.

Conclusion: A significant relationship between the adequate sampling results, the qualitative examination result, TI-RADS score and the needle type indicates the importance of needle type on the results of thyroid FNA. Using spinal (stylet) needles may reduce the number of non-diagnostic samples in fine needle aspiration of thyroid nodules and therefore, can have a better effect on the results of this method.
\end{abstract}

Keywords: Ultrasound-Guided Fine Needle Aspiration, Thyroid Nodule, Thyroid Diseases

\section{Background}

Thyroid nodules are solid, cystic or mixed lesions that form within the thyroid gland. Thyroid nodules are common and the vast majority of them are benign (noncancerous); however, a small proportion of thyroid nodules are proved to be malignant following biopsy or surgery (1). An appropriate systemic approach is required for evaluating thyroid nodules to avoid unnecessary surgeries. Thyroid fine needle aspiration (FNA) is currently the most effective and precise method for evaluating thyroid nodules. Various methods can be used for FNA of thyroid nodules (2). Al- though in some studies it has been shown that using ultrasound guide has no effect on the number of false-negative or non-diagnostic FNA results (3), ultrasound-guided aspiration is still the most efficient method (4). Despite false positive and negative results as other diagnostic tests, non-satisfactory sample results could be considered as the main limitation of ultrasound-guided aspiration $(4,5)$. Although various factors are defined to classify a sample as inadequate or non-diagnostic, the presence of six or more groups of 10 to 12 thyroid follicular cells on each of at least two slides is an acceptable criteria for a satisfactory result (6-8). In order to reduce the number of non-diagnostic 
samples, fine-needle aspiration by capillary action is suggested, and the operator's skill also plays an effective role in reducing the number of non-diagnostic samples (9). It has also been shown that spinal needles can reduce the number of non-diagnostic and inadequate samples (1012). Accordingly, this study aimed at comparing the results obtained from FNA of solid thyroid nodules using spinal (stylet) needle and simple needle in order to evaluate the role of the needle in FNA of the thyroid nodule.

\section{Objectives}

In this study, we compared the results obtained using spinal (stylet) needle and simple needle in thyroid nodule FNA according to the agreement between them and with the pathology results as the gold standard.

\section{Patients and Methods}

\subsection{Study Design and Patients}

The study population consisted of all patients who were referred to our university hospital through November 2018 to April 2019, who were candidates for FNA of thyroid according to the referring clinician decision. Patients who had a nodule with solid components and had no previously diagnosed thyroid cancer, were included in the study. Willingness of the patients to participate in the study and obtaining the informed consent from the subjects were also the inclusion criteria. The objectives of the study were explained to the participants. They were allowed to enter this study voluntarily. The patient's refusal to participate in the study does not interfere with the process of their treatment. Moreover, the proposal of the study was reviewed and approved by the Vice-Chancellor of Ethics Committee.

\subsection{FNA Method}

Following prep and drep of the patient's neck, 1 cc of $2 \%$ lidocaine was first injected using an insulin injection needle from the capsule of thyroid to the skin. Then sampling was performed by two different fine needles, a simple non-stylet 23 gauge needle ( $25 \mathrm{~mm}$ length) and a spinal 22 gauge needle with stylet (90 mm length) (Figure 1 ). Selection of the first needle for sampling was by random and one sampling was done with each needle. In our protocol, the needle was first entered into the nodule using ultrasound guidance (parallel to the transducer). For the spinal needle, the stylet was removed after entering the nodule. Then, the needle was attached to a 10-cc syringe. Sampling was performed through rapid, gentle and stabbing movements of the needle for several times (at least five times). If the color of the specimen appeared in the needle hub, the procedure was halted, otherwise sampling was repeated with 10-cc suction of the attached syringe. Sampling under suction, was also stopped if the specimen appeared in the hub or after several times of to and fro movements. Then the needle was withdrawn. In the next step, the needle contents were placed on clean slides, smeared and fixed. After sampling by first randomly selected needle, the procedure was repeated using the second needle. During the needle production to the nodule and sampling, the needle was under real-time ultrasound surveillance.

The samples were marked as A (simple needle) and B (spinal needle) and provided to the pathologist for a blind examination. All samples were obtained by an expert radiologist in FNA of the thyroid with more than 10 years' experience and the pathology examination was also performed by a pathologist expert in thyroid gland diseases. Quantitative and qualitative criteria were used to investigate the FNA results.

\subsection{Pathological and Statistical Evaluation}

At least 6 cell clusters with at least 10 follicular cells were considered as a quantitative criterion for an appropriate sample (6-8). According to the qualitative criteria, the expert pathologist selected the most appropriate sample out of two samples, which were blind to them. At the end, a comparison was made using statistical tests for the rate of cancer diagnosis in the samples, the rate of insufficient samples, the rate of blood contamination and the rate of sample appropriateness between two needles. Qualitative results (according to expert pathologist opinion and qualitative criteria mentioned before), pathology results (according to histological and pathological reports) and relation between Thyroid imaging reporting and data system (TI-RADS) score and FNA result (according to radiological and pathological reports) were compared between simple needle and spinal needle. Blind analysis was performed using SPSS Statistics for Windows, version 16.0 (SPSS Inc., Chicago, Ill., USA) by chi-squared test at a significance level of $\mathrm{P}<0.05$. Due to the better results obtained by spinal needle in biopsy, the study was not considered ethically appropriate to be continued after 156 patients.

\section{Results}

According to the inclusion and exclusion criteria, a total of 156 patients and 312 samples were examined. However, due to better results obtained by stylet (spinal) needle and as a result, a better diagnosis of cancer, the study was not considered ethically appropriate to be continued. The demographic information of the patients is shown in Table 1. 


\begin{tabular}{|c|c|}
\hline Parameters & Values $^{\mathrm{a}}$ \\
\hline Age, $y$ & $48.63 \pm 13.42$ \\
\hline \multicolumn{2}{|l|}{ Sex } \\
\hline Male & $21(13.48)$ \\
\hline Female & $135(86.42)$ \\
\hline Nodule size, $\mathrm{cm}$ & $1.23 \pm 0.39$ \\
\hline
\end{tabular}

${ }^{\mathrm{a}}$ Values are expressed as mean \pm SD or No. (\%).

According to the quantitative results in analysis of the samples, the results of FNA with needles A and B were reported unsatisfactory in 11.51 (18 subjects) and 3.84 (six subjects), which was statistically significant $(P=0.01)$. In addition, based on qualitative results of pathology, in $63.46 \%$ (99 subjects) of the participants, the sample obtained by needle B was introduced as a better sample by the pathologist, in comparison with $36.44 \%$ (57 subjects), which was statistically significant $(\mathrm{P}<0.001)$. Qualitative and quantitative results are shown in Table 2. Cancer was diagnosed in the samples yielded by needles B and A, 9.56 and 7.66, respectively, which was not statistically significant $(\mathrm{P}=0.54)$. Pathology result of both methods is summarized in Table 3. Considering TI-RADS, different groups of susceptibility show better results by using spinal needle. Data is summarized in Table 4.

Fortunately, no procedure-related complications, including hematoma or infection were reported.

\begin{tabular}{|c|c|c|}
\hline Results & Frequency (\%) & Pvalue \\
\hline Quantitative & & 0.01 \\
\hline \multicolumn{3}{|l|}{ Simple needle } \\
\hline Unsatisfactory & $18(11.41)$ & \\
\hline Satisfactory & $138(88.49)$ & \\
\hline \multicolumn{3}{|l|}{ Spinal needle } \\
\hline Unsatisfactory & $6(3.76)$ & \\
\hline Satisfactory & $150(96.24)$ & \\
\hline Qualitative & & $<0.001$ \\
\hline \multicolumn{3}{|l|}{ Sample of choice } \\
\hline Simple needle & $57(36.44)$ & \\
\hline Spinal needle & $99(63.46)$ & \\
\hline
\end{tabular}

Abbreviation: FNA, fine-needle aspiration.

\begin{tabular}{|c|c|}
\hline Needle type, pathology & Frequency (\%) \\
\hline \multicolumn{2}{|l|}{ Simple needle } \\
\hline Follicular adenoma & $126(80.83)$ \\
\hline Cancer & $12(7.66)$ \\
\hline Non-diagnostic & $18(11.51)$ \\
\hline \multicolumn{2}{|l|}{ Spinal needle } \\
\hline Follicular adenoma & $135(86.51)$ \\
\hline Cancer & $15(9.56)$ \\
\hline Non-diagnostic & $6(3.84)$ \\
\hline
\end{tabular}

Abbreviation: FNA, fine-needle aspiration.

\section{Discussion}

Ultrasound is regarded as the most accurate method to examine the thyroid, parathyroid and cervical lymph nodes. Using ultrasound in thyroid examination has increased the diagnosis of nodules undetectable in clinical examination. However, only a small number of these nodules are malignant. Significant progress has been made in the management of thyroid nodules using ultrasoundguided FNA, and it is considered as the most reliable and cost effective technique to differentiate benign thyroid nodules from suspicious and malignant nodules (13). Unfortunately, inadequate sampling is one of the limitations

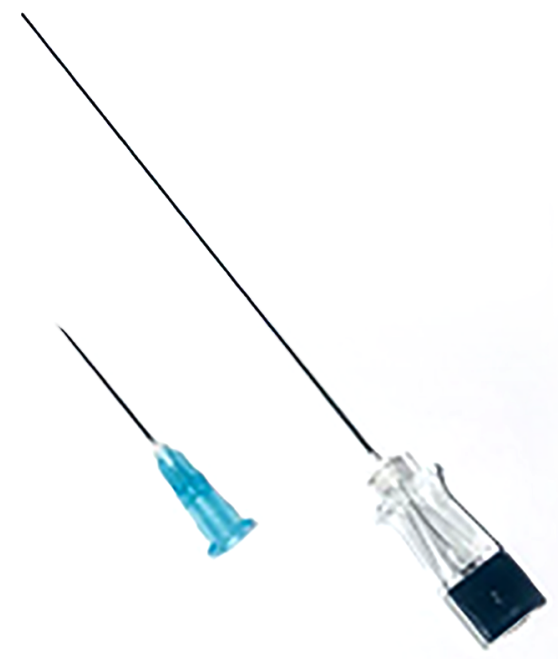

Figure 1. Simple non-stylet 23 gauge needle (25 mm length) on the left and spinal 22 gauge needle with stylet ( $90 \mathrm{~mm}$ length) on the right 


\begin{tabular}{|c|c|c|c|c|c|c|}
\hline \multirow{2}{*}{ Risk } & \multirow{2}{*}{ Points } & \multirow{2}{*}{ Samples } & \multirow{2}{*}{$\begin{array}{c}\text { Qualitative } \\
\text { pathology results }\end{array}$} & \multicolumn{2}{|c|}{ Needle type } & \multirow{2}{*}{ Pvalue } \\
\hline & & & & Simple needle & Spinal needle & \\
\hline \multirow{2}{*}{ Mildly suspicious } & \multirow{2}{*}{3} & \multirow{2}{*}{68} & Unsatisfactory & 4 & 1 & \multirow{2}{*}{0.04} \\
\hline & & & Satisfactory & 64 & 67 & \\
\hline \multirow{2}{*}{ Moderately suspicious } & \multirow{2}{*}{$4-6$} & \multirow{2}{*}{174} & Unsatisfactory & 9 & 4 & \multirow{2}{*}{0.002} \\
\hline & & & Satisfactory & 165 & 170 & \\
\hline \multirow{2}{*}{ Highly suspicious } & \multirow{2}{*}{$\geq 7$} & \multirow{2}{*}{70} & Unsatisfactory & 5 & 1 & \multirow{2}{*}{0.02} \\
\hline & & & Satisfactory & 65 & 69 & \\
\hline
\end{tabular}

Abbreviations: FNA, fine-needle aspiration; TI-RADS, thyroid imaging reporting and data system

of this technique. Cappelli et al. showed that Yale spinal (YS) significantly reduced the number of non-diagnostic samples, and as a result, decreased the direct and indirect costs (12). In addition, another study by Cappelli et al. indicated that stylet needles could improve the diagnosis and also reduce its costs by $14 \%$ (11). Moreover, Cappelli et al. demonstrated that stylet needles significantly improve the percentage of adequate sampling in FNA of complex thyroid nodule (10). In the present study, we showed that spinal needles significantly increase the percentage of adequate sampling $(\mathrm{P}=0.009)$. By using stylet needles (similar to the spinal needle used in this study), no sample can enter the needle while crossing tissues in the path. The sample can only enter the needles after reaching the nodule and leaving the stylet from the thyroid nodule. Accordingly, stylet needles are more effective in the FNA of the thyroid nodule. Also, it is notable that in our study, using spinal needles, more than three cancer cases were diagnosed, indicating the importance of needles. However, this needs to be confirmed by clinical trials with a larger sample size.

In some studies, it has been shown that nodule ultrasound patterns can also affect the number of false-negative results (14), so at the present study, only solid nodules were subjected to FNA. In a study conducted by Cappelli et al., sampling was only done using complex nodules (10). Accordingly, in the present study, all solid nodules were studied to obtain more comprehensive results. However, to achieve more comprehensive outcomes, it is suggested to study the relationship between nodule characteristics, such as the TI-RADS and American Thyroid Association (ATA) criteria and the results of these two methods in future studies.

Proper prep and drep can be observed using spinal needles in biopsy, so less infection and complications are resulted. In addition, due to administration of lidocaine and anesthesia in this method, the patient feels less pain, is more psychologically prepared and will cooperate more with the treatment team; therefore, sampling in this method is more accurate. However, these two advantages should be confirmed by more comprehensive and standardized studies.

Another difference between the spinal needle and the simple needle is the length of the needle $(25 \mathrm{~mm}$ versus $90 \mathrm{~mm}$ ) that results in a larger sample size and volume in spinal needle FNA and therefore, could have a better effect on the results of this method.

In this study, spinal needles were used as the second method of sampling in some cases, so the changes in the tissues made by simple needle could affect the results, which could be considered as one of the limitations. Other limitations of this study could be lack of physical and human resources, as well as lack of a system for accurate recording and following-up of the patients, which resulted in the prolonged study and loss of some samples.

In conclusion, despite these limitations, a significant relationship between adequate sampling results, qualitative examination result and needle type indicates the importance of needle type on the results of thyroid FNA. It seems there is no increased risk of complications by using spinal needles in thyroid nodule FNA. Using spinal needles may reduce the number of non-diagnostic samples in thyroid nodule fine needle aspiration and therefore, could have a better effect on the results of this method.

\section{Footnotes}

Authors' Contributions: Study concept and design: Alireza Aziz Ahari and Navid Khatibi Moghadam; acquisition of data: Mohammadreza Khaleghi, Navid Khatibi Moghadam, and Mohammad Ali Mohammadi Vajari; analysis and interpretation of data: Mohammadreza Khaleghi and Hassan Hashemi; drafting of the manuscript: Mohammad Ali Mohammadi Vajari and Alireza Aziz Ahari; critical revision of the manuscript for important intellectual content: Alireza Aziz Ahari and Mohammad Ali Mohammadi 
Vajari; statistical analysis: Mohammadreza Khaleghi, Mahmoud Parvin, and Alireza Aziz Ahari; administrative, technical, and material support: Alireza Aziz Ahari and Mohammad Ali Mohammadi Vajari; study supervision: Alireza Aziz Ahari, Hassan Hashemi, and Mahmoud Parvin.

Conflict of Interests: Authors have no conflict of interests.

Ethical Approval: Willingness of the patients to participate in the study and obtaining informed consent from the subjects were also inclusion criteria. The objectives of the study were explained to the participants. They were allowed to enter this study voluntarily. This study did not impair health services. Also, the proposal of the study was reviewed and approved by the Vice-Chancellor of Ethics Committee of Iran University of Medical Sciences (IR.IUMS.9611282058).

Funding/Support: The authors received no financial support for the research, authorship, and/or publication of this article.

Patient Consent: Informed consent was received from all patients.

\section{References}

1. Parsa AA, Gharib H. Epidemiology of thyroid nodules. Thyroid nodules. Humana Press; 2018. p. 1-11. doi: 10.1007/978-3-319-59474-3_1.

2. Wu H. Techniques of thyroid fine-needle aspiration. Thyroid FNA cytology. Singapore: Springer; 2019. p. 483-9. doi: 10.1007/978-981-13-18979_64.

3. Choong KC, Khiyami A, Tamarkin SW, McHenry CR. Fine-needle aspiration biopsy of thyroid nodules: Is routine ultrasound-guidance necessary? Surgery. 2018;164(4):789-94. doi: 10.1016/j.surg.2018.04.047. [PubMed: 30072248].
4. Wang HH. Reporting thyroid fine-needle aspiration: Literature review and a proposal. Diagn Cytopathol. 2006;34(1):67-76. doi: 10.1002/dc.20400. [PubMed:16355378].

5. Gharib H. Fine-needle aspiration biopsy of thyroid nodules: Advantages, limitations, and effect. Mayo Clin Proc. 1994;69(1):44-9. doi: 10.1016/s0025-6196(12)61611-5. [PubMed: 8271850].

6. Cibas ES, Ali SZ. The 2017 bethesda system for reporting thyroid cytopathology. Thyroid. 2017;27(11):1341-6. doi: 10.1089/thy.2017.0500. [PubMed: 29091573].

7. Cibas ES, Ali SZ. The bethesda system for reporting thyroid cytopathology. Thyroid.2009;19(11):1159-65. doi:10.1089/thy.2009.0274. [PubMed: 19888858].

8. Crippa S, Mazzucchelli L, Cibas ES, Ali SZ. The Bethesda System for reporting thyroid fine-needle aspiration specimens. Am J Clin Pathol. 2010;134(2):343-4. author reply 345. doi: 10.1309/AJCPXM9WIRQ8JZBJ. [PubMed: 20660341].

9. De Fiori E, Rampinelli C, Turco F, Bonello L, Bellomi M. Role of operator experience in ultrasound-guided fine-needle aspiration biopsy of the thyroid. Radiol Med. 2010;115(4):612-8. doi: 10.1007/s11547-010-0528-x. [PubMed: 20177981].

10. Cappelli C, Pirola I, Castellano M, Gandossi E, De Martino E, Delbarba A, et al. Fine needle cytology of complex thyroid nodules. Eur J Endocrinol. 2007;157(4):529-32. doi: 10.1530/EJE-07-0172. [PubMed: 17893269].

11. Cappelli C, Pirola I, Gandossi E, De Martino E, Agosti B, Castellano M. Fine-needle aspiration cytology of thyroid nodule: Does the needle matter? South Med J. 2009;102(5):498-501. doi: 10.1097/SMJ.ob013e31819c7343. [PubMed:19373168].

12. Cappelli C, Tironi A, Mattanza C, Cumetti D, Agosti B, Gandossi E, et al. Cost-effectiveness of fine-needle-aspiration cytology of thyroid nodules with intranodular vascular pattern using two different needle types. Endocr Pathol. 2005;16(4):349-54. doi: 10.1385/ep:16:4:349. [PubMed: 16627921].

13. Castro MR, Gharib $H$. Thyroid fine-needle aspiration biopsy: Progress, practice, and pitfalls. Endocr Pract. 2003;9(2):128-36. doi: 10.4158/EP.9.2.128. [PubMed: 12917075].

14. Ahn HS, Na DG, Baek JH, Sung JY, Kim JH. False negative rate of fineneedle aspiration in thyroid nodules: Impact of nodule size and ultrasound pattern. Head Neck. 2019;41(4):967-73. doi: 10.1002/hed.25530. [PubMed: 30740835]. 